\section{An Aduress}

on

\section{THE SIGNS OF EARLY DISEASE OF THE THYROID GLAND.}

Delivered at a Combined Meeting of the Burnley and District Medico-Ethical Society, and Burnley Division of the British Medical Association.

By GEORGE R. MURRAY, M.A., M.D.CAMb., Hon. D.C.L.DuRH., F.R.C.P.,

CONSULTING PHYSICIAN TO THE ROYAL VICTORIA INTFIRMARY, NEWCASTLE; PROFESSOR OF SYSTEMATIC MEDICINE, VICTORIA UNIVERSITY OF MANCHESTER; PHYSICIAN TO THE MANCHESTER ROYAL INFIRMARY.

IN many diseases which develop gradually and run a chronic course the earliest signs of ill-health are apt to be overlooked. The textbooks, as a rule, describe a disease as it appears in its typical form, while insufficien't attention is paid to the symptoms of the early or mild forms of the malady. It thus happens that a disease may have made considerable progress before it is recognized, and so valuable time is lost, during which suitable treatment might have been applied.

I propose, therefore, to-day to draw your attention to some of the signs of early thyroidal disease, which, though by no means rare, are not as well known as they might be.

In diseases of the thyroid gland symptoms of two kinds may occur. In those in which the gland is increased in size there may be local symptoms due to the enlargement itself. These are mechanical in origin, and are the result of the pressure exerted by the goitre upon neighbouring structures in the neck. The other symptoms are due to variations in the functional activity of the gland induced by the structural changes which occur in it.

When the various pathological changes to which the thyroid gland is liable are fully developed the symptoms are so marked and striking that the diagnosis presents no difficulty. The various forms of goître are readily recog nized as such, the real difficulty being not to determine whether the tumour is a goitre or not but to distinguish between the different varieties of goitre which may occur. A well-marked case of exophthalmic goître, of myxoedema, or of cretinism is readily diagnosed, provided the observer is familiar with the general aspect of the disease. On this occasion we may with advantage consider some of the early symptoms presented by these diseases by which we may recognize them at the earliest possible moment and so lose no valuable time in applying the most appropriate treatment. The importance of this is twofold. First, one finds that patients in the earlier stages of thyroida disease may go about for a considerable period without the true nature of the disorder being recognized; secondly, the value of early treatment is so great, both in diminishing the period of ill-health and in obtaining a better result than when it is deferred until the disease has become fully established.

Some of these cases with slight symptoms are in an early stage of a more severe attack which will follow if treatment is not successful in checking their further development. : Others are mild types of the disease, and may for long remain stationary in this condition. 'Such cases, both of myxoedema and Graves's disease, have been described by Continental writers as formes frustesan unnecessary term, as they are simply mild cases such as we find in so many other maladies.

As the function of the thyroid gland is to supply an internal secretion which escapes by way of the lymphatics into the blood stream, by which it is distributed to all parts of the body, diseases of the thyroid may be divided into three classes, according to the manner in which this function of the gland is influenced by them. Thus some diseases have no appreciable effect upon the secretory function of the gland ; others lower or abolish it according to their severity, and in yet a third class it is greatly increased and possibly perverted as well.

As examples of the first class may be cited uncomplicated acute thyroiditis, simple parenchymatous goître, and many cases of adenomatous or cystic goitre. The second class is illustrated by cretinism and myxoedema, and the third by exophthalmic goitre and those cases of goitre in which the "goitre-heart " is present.

Simple Parenchymatous Goître.

The normal thyroid gland is subject to variations in volume, as it may becomo temporarily enlarged during menstruation or pregnancy, subsiding afterwards to its usual size. Such enlargements are physiological, and occur in response to some temporary stimulus or demand for an increased functional activity. In many cases, however, a simple enlargement of this kind tends to go on increasing in size and becomes pathological. This occurs most frequently in adolescents and young adults, and much more frequently in women than in men. In the early stages the swelling is apt to escape notice unless the thyroidal region is inspected and palpated in the course of a routine medical examination. As the thyroid grows the total circumference of the neck is increased, and the gradual tightening of the collar may first draw attention to the goitre. On inspection the front of the neck looks full and rounded, and the -skin is often marked by the pressure of the collar stud. If the patient be made to swallow a mouthful of water the swelling is seen to rise and fall again during the act of deglutition. This movement of the swelling is characteristic of all enlargements of the thyroid gland unless it is fixed by adhesions, or, as in the case of malignant goittre, by the extension of the disease through the capsule into the surrounding parts. During this movement the horseshoe shape of the uniformly enlarged gland is often clearly to be seen. On palpation the swelling will be found to take the same shape as the thyroid gland, though it will be several times as large. It is firm and elastic in consistence, and there is no tenderness on pressure. No thrill can be felt, nor is any murmur as a rule audible on auscultation over it. When the goitre has reached a larger size, the veins overlying it may be enlarged and visible; there may be a sense of fullness in the neck and some difficulty in breathing. This, however, is much more common in adenomatous and cystic goîtres than in the form under consideration.

This simple parenchymatous goître may be distinguished from exophthalmic goître by the absence of tachycardia and: other symptoms of Graves's disease, and from enlargement due to the presence of adenomata or cysts by its symmetrical shape, though of course the two forms of enlargement may be combined in the same case.

As this form of goitre is a simple hypertrophy which takes place in response to a demand for increased activity, it is analogous to the hypertrophy of the mammary gland which takes place during pregnancy and lactation. The treatment is therefore on rational lines, and consists in supplying the increased amount of thyroidal secretion which is required from another source. When this is done the gland gradually diminishes in size. If the treatment is commenced at an early stage the gland may return to its normal size. If the hypertrophy has been present for some time the result may not be so good, but a marked reduction in size may be expected in many cases. As an illustration of this class of case I may refer briefly to the case $^{1}$ of a girl, aged 13, who was under my care at the Newcastle Royal Infirmary, in which there was a considerable goittre which caused some difficulty in breathing. The total circumference of the neck was $16 \frac{1}{2}$ in., and after treatment for eleven weeks it was reduced to 13 in., and the symptoms due to the goitre were removed. In this and other similar cases the question of an operation for the removal of a portion of the goitre to relieve the dyspnoea had been considered, but was obviated by the treatment.

As a general rule, when a goitre is unilateral, the enlargement is due to an adenoma or cyst in one lobe. The size of an adenoma or a cyst would not be affected by thyroid extract. I have, however, seen one interesting case which was sent to me by Dr. A. Smith, of Whickham, in which one lobe alone was enlarged. I regarded: this enlargement as being due to an adenoma, and advised its removal, as there were signs of pressure. The patient, however, refused operation, and therefore we decided to try the effect of thyroid treatment. To our surprise, the enlarged lobe steadily diminished in size, and the patic nt was reliered of her symptoms. This case suggests that a $[25$ I I ] 
unilateral goitre may in some cases be due to a simple hypertrophy, and that it is worth while to try the effect of this treatment if there is any donbt as to the cause of the enlargement.

In cases of simple goitre it generally suffices to give from 3 to 5 grains of dry thyroid powder in a tablet each night. In some cases it is advisable to give this dose two or three times daily, provided no marked acceleration of the pulse occurs. If there is no distinct reduction in the size of the goitre after treatment for a month or six weeks, there is nothing to be gained by continuing it further. If there is a decided reduction, it is advisable to continue the treatment for three or four months, so as to obtain its full benefit, and to resume it for a month occasionally if there is any sign of recrudescence.

If goitre is at all prevalent in the district the patient should drink no water unless it has previously been boiled or distilled, The local application of iodine, especially in the form of the red iodide of mercury ointment, is also useful.

\section{MYXOEDEMA.}

We may pass on now to the consideration of the mild type of myxoedema which results from a partial fibrosis of the thyroid gland, which occurs not uncommonly in women between 40 and 50 years of age. When the fibrosis is complete, a fully-developed case of myxoedema results. In arriving at this advanced stage of the disease, the symptoms are at first slight. I believe, however, in some cases that the fibrosis of the thyroid is only partial for a long period. In some it possibly never progresses beyond this stage. Thus, we occasionally come across these mild eirly cases of myxoedema which have been neither recog nized nor treated, the result being a prolonged period of ill. health for the unfortunate sufferer, which she might have bean saved if the disease had been diagnosed and treated at its commencement.

The symptoms which result from partial thyroidal fibrosis usually come on very gradually, between 40 and 50 7ears of age, and are not infrequently mistaken for those which so commonly occur in women during. the menopause. There is a gradual loss of mental and physical energy, so that every action appears to require a special effort. The result is that the patient's sphere of action becomes more and more restricted. Such patients say they take no pleasure in life and have lost interest in their surroundings. They are apt to shun the society of others and to become depressed and melancholy at times. If by force of will a normal mode of life is continued they will tell you this is only accomplished by a considerable mental effort and expenditure of energy. The memory is often defective, especially for names and for recent events. Slight visua hallucinations are very common. They are rarely com plained of, bat a clear account of them may be obtained on carefully interrogating the patient. In these slight cases the hallucinations are not, as a rule, so clearly defined as they are in some advanced cases, but take the form of some ill-defined object, indistinctly seen "out of the corner of the eye," like a mouse rapidly crossing the room. Sensations of cold are often complained of, although the temperature of the body may be scarcely, if at all, below normal. The facial appearance is somewhat altered. The cheeks become rounded and fuller, and a central pink flush appears. This change may impart a fictitious appearance of improved health and nutrition, which is liable to be mistaken for such by the friends of the patient, who imagine that she is getting stout and rosy. A careful inspection will, however, show that there is also a small amount of swelling of the eyelids, which has translucent appearance like the swelling due to a slight subcutaneous oedema, but does not "pit" on pressure. The skin of the face may be rather pale and waxy in appearance, or it may have a pale yellow tinge. The lips are rather swollen and the mucous membrane pale. The gyebrows may be rather scanty, and the forehead is often transversely wrinkled.

The subcutaneous swelling on the body generally is not sufficient to attract the patient's attention, though it may be found on careful examination. In the supraclavicular region the swelling may be quite distinct. The hands are somewhat altered in appearance, the back of the hand being rounded and swollen. The feet often show a similar condition, though the swelling may not be sufficient to necessitate the use of a larger size of boot, as in the more fully-developed stages of the malady. The skin is rather dry; on the backs of the hands it may be smooth if there is much swelling, or it may be loose and wrinkled, like that on the hand of a washerwoman. A fine desquama. tion takes place, especially on the legs, the results of which are clearly seen, on removing a pair of black stockings and turning them inside out, as a fine white powder. The hair may partially come out, though its texture may not be appreciably altered.

Such are the main symptoms produced by early thyroidal fibrosis, easily recognized when looked for, but otherwise easily passed over. In doubtful cases a careful examination of the thyroidal region of the neck may show that the gland is diminished in size. As already mentioned, these symptoms are liable to appear at the time of the menopause. When the ovary atrophies at the same time as the thyroid gland, the symptoms of the menopause, such as a feeling of pressure on the top of the head, hot flushes and sweats, may occur in addition to those which are due to the thyroidal fibrosis. The nervous symptoms in each case are somewhat similar. A careful examination of the patient for the slight signs of myxoedema will, however, enable a correct conclusion to be drawn as to the cause of the symptoms complained of.

There is a condition of lipomatosis which $I$ have seen in several cases which closely resembles a slight form of myxoedema, which has not as yet received adequate recognition. In this there is an elastic subcutaneous swelling like that which we see in myxoedema, bat differing from it in distribution, The skin is not dry as it is in true myxoedema. The hair and nails are unaffected. The swelling is generally more localized than in myxoedema. One case which appeared to be of this type occurred in a lady of 31 . There were wellmarked swellings in the supraclavicular region, over the deltoids, over the hips and upper gluteal region. The swelling was greater on the right side of the body than the left. The circumference of the right arm at the level of the insertion of the deltoid was 13 in., of the left $12 \frac{1}{3}$ in. The circumference of the middle of the right thigh was $22 \frac{1}{2}$ in., of the left $20 \frac{1}{2}$ in. In these cases the swelling has no sharp edge, but gradually shades off, and it is quite painless. In addition to the situations already mentioned, it may be present on the backs of the hands. As a rule the face is not affected; if it is the swelling is only in the cheeks, which have no central flush, and there is no swelling of the eyelids as in myxoedema. When the sweiling covers a considerable area, as it does on the outside of the thigh, the skin is dimpled when it is pinched up between the tinger and thumb. The final test is the action of thyroid extract. It will be found that even after taking two or three times as much thyroid extract as would suffice to relieve a true myxoedema in the course of a month, these swellings are only slightly, if at all, diminished in size. They appear to consist of irregular deposits of subcutaneous fat, and are possibly more closely allied to the swellings which occur in adiposis dolorosa, from which, however, they differ in size and in the absence of all pain and tenderness.

The treatment of these cases of mild myxoedema is simple. Thyroid extract should be given in doses of 5 minims of liquor thyroidei each night for a month. If decided improvement has then taken place this dose may suffice, if not, it is advisable to increase it up to 10 minims, and to continue this dose until all the symptoms have disappeared. The permanent dose will be from 5 to 7 minims, for it must be clearly understood, and also explained to the patient at the outset, that the treatment must be continued for the rest of her life. If, later on, the partial fibrosis becomes total it will be necessary to increase the permanent dose to 10 minims each night. This amount of the extract appears to correspond to the daily output of secretion from an average normal gland. This statement is based on the fact that my first case of myxoedema, in which $I$ believe there is now no active portion of the gland left, has been kept quite free from myxoedema for more than seventeen years by this dose taken six nights in the week.

\section{Exophthalmic Goître.}

We have now to consider the effects of hypersecretion of the thyroid gland, just the opposite condition to that 
which obtains in myxoedema. The result of excessive secretion is best seen in a typical case of exophthalmic goittre. The microscopical appearance of the enlarged thyroid gland exhibits changes which indicate that there is an enormous increase of functional activity. Fullydeveloped exophthalmic goitre is so characteristic that no description is required. The goître, exophthalmos, rapidly-beating heart, with nervousness, tremors of the hands, and emaciation, make up a clinical picture which can be readily recognized. It is, however, to the less -obvious cases that I wish more particularly to draw your attention. In the first place I may remind you that in more than a quarter of all the cases of definite Graves's disease one symptom, and that the most obvious of all, the exophthalmos, is absent. Thus in 170 of my own cases ${ }^{2}$ exophthalmos was present at some stage of the disease in 123 , but in 47 , or more than 25 per cent., there was no -exophthalmos at all. Why this remarkable sign is present in one case and yet absent in another which otherwise has similar symptoms cannot at present be explained. The whole pathology and causation of the exophthalmos is still obscure. Of the various explanations of it which have been given, the only one which is tenable is that which attributes it to an abnormal development of the retro-bulbar fat in the orbit. At an autopsy this mass of fat presents a striking appearance, and may be seen even in cases in which there is such a degree of general emaciation that hardly any fat is visible in other parts of the body. The fat from one orbit alone in one of my cases weighed $\frac{1}{2}$ oz. A simple experiment will show how such a mass of fat produces exophthalmos. The head of a dead sheep or smaller animal, such as a guinea-pig, will serve the purpose quite well. The upper part of the skull and the brain are removed so as to expose the optic foramen. A small trocar and cannula are pushed through the optic foramen alongside the optic nerve until the orbit is reached. The trocar is withdrawn, and a warm syringe filled with melted paraffin or agar-agar to which some colouring matter has been added is attached to the cannula. The fluid is then injected into the orbit behind the eyeball, which gradually begins to protrude, and any degree of exophthalmos can be produced according to the amount of fluid injected. Later the orbit can be opened and the distribution of the solidified agar-agar examined. The exophthalmos thus persists after death, which it would not do if it was caused by vascular congestion or spøsm of Müller's muscular fibres.

Exophthalmos is so striking a symptom that when present the diagnosis is easy. It is, however, to the cases in which this symptom is absent that I wish to direct your attention. When there is no actual exophthalmos there may be a rather staring expres:sion in some cases, which is due to a slight spasm of the elevator of the upper eyelid (Stellwag's sign). This may be either constant or intermittent. When it is persistent there is a notable absence of blinking, the palpebral fissure is rather wider than it should be, and the effect is to give a rather startled expression. When it is temporary it may only appear for a moment when the eyes are suddenly directed in some new direction. The patient will probably come to you complaining of palpitation. This may only be felt from time to time, or it may be continuous. If always present it is easily aggravated by any exertion or excitement. Disagreeable throbbing, especially in the carotid arteries, is often mentioned. The pulse will be found to be frequent100 or very often 120 , or even 130 or 140 . If the patient is examined when lying in bed the pulse will be less frequent by 10 or 20 beats a minute. Apart from infective diseases and organic disease of the heart, Graves's disease is the commonest cause of persistent tachycardia. In fact, in all cases, apart from cardiac and infective maladies, in which there is a frequent pulse of 90 or more, it is most important to look carefully for other signs of exophthalmic goitre. Many of these cases are described as examples of paroxysmal or persistent tachycardia and their true nature overlooked. This frequent pulse is such a constant symptom that in 178 of my cases of exophthalmic goitre in which the frequency was recorded, in only 4 was the pulse between 90 and 100, while in 174 it was between 100 and 200. In some of these slight or early cases palpitation may not be complained of, and it is only on examination that the high frequency of the pulse is discovered.
On examination the heart is found to be beating more or less violently and the cardiac impulse can be both seen and felt over a larger area than usual. In these mild cases of the disease there is generally no dilatation or other change in the heart itself. The action of the heart is precisely similar to that we often find in a healthy but nervous young man who presents himself for examination for life assurance or who is undergoing any other mental strain or has just taken violent exercise. If the tachycardia is the only symptom present and the thyroid gland is not enlarged, we may conclude that it is not a case of Graves's disease. For example, I recently saw a lady, aged 52, who had suffered from palpitation for four years. The palpitation came on during an attack of influenza. The pulse was 120 sitting, 104 lying down. The thyroid was normal in size, there were no ocular symptoms, no definite tremor and the weight was increasing. The heart was normal in size and there was no valvular disease. Such a case must be regarded as one of simple postinfluenzal tachycardia.

The enlargement of the thyroid gland is generally slight. The gland may be only twice or thrice its normal size, so that the swelling may not even be noticed by the patient at all. It is necessary, therefore, in these cases to make a carefal examination of the front of the neck to determine whether there is enlargement or not. It is important while doing this to make the patient swallow a mouthful of water, as in some cases the gland lies rather low down in the neck, and a slight enlargement is more readily detected during the upward movement which accompanies deglutition. The enlargement is uniform and painless. A very notable feature in these cases is the mental condition. The general characters of the mental state in the fully developed disease are well known. In the slight cases also the mental condition may be quite charac. teristic. There is a condition of irritable weakness, in which a desire to perform many active duties is accom panied by an inability to accomplish them without inducing a disproportionate amount of fatigue. Such patients are anxious to undertake useful work, but are very fussy and nervous in doing it. They feel uncomfort. able. when in a crowded assembly, and are often obliged to leave early. They are restless, and find it difficult to continue any single occupation for long. There is a remarkable tolerance of cold and sensitiveness to heat, a result, doubtless, of the increased oxidation going on in the tissues. This condition of general nervousness often shows itself in the presence of strangers.

The characteristic fine tremor of the hands is usually present, though it may be necessary to look closely to see it. This tremor was present in 163 of my 180 cases. It was absent in 7, and was not noticed in 10. Weakness of the legs and a feeling as if they would give way in walking may be complained of in these mild cases.

No case should, then, be regarded as one of Graves's disease unless the pulse is 90 or more a minute, and there is either. slight exophthalmos, some enlargement of the thyroid, or tremor as well. If two or three of these symptoms are present, the case should be regarded as a mild case of Graves's disease.

There is a special variety of Graves's disease which is generally described as secondary, which may occur in a mild form. As I have already mentioned, in cases of simple goître of various kinds there is, as a rule, no evidence of hypersecretion, and so the symptoms are purely local. In some cases of goître, even of some standing, however, the secreting activity becomes increased. This may be so marked that the symptoms of Graves's disease are fully developed, and there is no difficulty in recog. nizing that "we have to deal with a distinct case of secondary Graves's disease. Fourteen of my 180 cases were of this type. In 2 of them the goittre had been present for thirty years, and in others for various periods of from three to twenty-five years before symptoms of hypersecretion developed.

There are, however, cases of goitre in which tachycardia appears alone without other -symptoms. These cases have been described by Continental writers as examples of "goitre-heart," so it is well to recognize the existence of these cases, especially in view of any operative treatment, as there is more risk when these signs of cardiac disorder are present than when they are absent altogetber. The pathology of this condition is probably the same as 
that of Graves's disease, the symptoms being due to excess or change in the character of the thyroidal secretion.

It is also well to remember that if for any reason a patient has been taking large doses of thyroid extract for a long period a condition of thyroidism may be produced in which many of the symptoms of Graves's disease develop-such as tachycardia, tremor, nervousness, sweating, and emaciation.

Time will only permit me to allude very briefly to the treatment of these slight cases of exophthalmic goître. As in fully developed cases, we must enforce a sufficient amount of mental and bodily rest according to circumstances. A liberal diet, which includes two or three pints of milk, is required to compensate for the increased metabolism. A mild faradic current applied to the neck for two or three hours daily is often most beneficial. A prolonged course of arsenic in small doses, with or without the addition of tincture of convallaria, is also useful in most of the cases. On no account should thyroid extract be given in these cases, as it naturally only aggravates the symptoms.

REFERENCEs.
1 Edinburgh Medical Journal, August, 1900. 2 Lancet, November 11th, 1905.

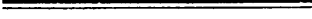 \\ Hemarkz}

\author{
oN
}

THE NATURE AND TREATMENT

OF

\section{PARENCHYMATOUS GOT'TRE. \\ By FRANCIS L. A GREAVES, F.R.C.S.,} SURGEON TO THE DERBYSHIRE ROYAL INFIRMARY.

UNDER the term "goitre" several varieties of enlargement of the thyroid gland are embraced, and in classifying them great difficulty is felt at the outset from the fact that the varieties are not very distinct.

Part of the gland may show changes characteristic of one class, while another part may display all the features of a goitre of another class. English pathologists regard parenchymatous goitre as distinct from adenoma of the thyroid, but the German schools do not make this distinction. But there is much to recommend the English classification from the point of view of the practical surgeon.

The normal thyroid gland consists of a vascular fibrous stroma, including in its meshes rounded follicles or acini which either possess a central lumen or are filled entirely with epithelial cells. The acini are lined with a single layer of cubical epithelium without basement membrane, and the lumen may be either clear or filled with colloid material.

\section{Parenchymatous Goître.}

In parenchymatous goitre there is glandular proliferation which results in the production of clusters of cells or of gland-like acini lined with epithelium, which may be somewhat irregular and flattened compared with the normal gland. Both varieties may be seen in the same specimen. The cut surface of a fresh goitre shows to the naked eye a reddish or dark brown colour ; in parts where many of the acini contain masses of colloid material the section has a translucent honey-like appearance. All the tissue elements in these goitres are increased in amount.

The patient presents a smooth, full neck, reminiscent, when present to a small extent, of Burne-Jones's paintings. This condition is more common in females than in males, and generally commences about the age of puberty. It is at first quite painless, giving rise to no discomfort. The consistency of such a gland is rather soft and has a somewhat elastic feeling on palpation. It is movable on swallowing.

It frequently enlarges during the menstrual period, and the patient sometimes complains of a slight sensation of suffocation at that time. The voice is not altered and no laryngoscopic changes are to be seen. In many of these cases I have found great hypertrophy of the tousils, and removal of these seems to have some influence in producing diminution in the size of the gland; possibly this may be due to the improvement of the patient's general condition.

This mild variety of parenchymatous goitre, which is exceedingly common in Derby and Derbyshire, often subsides gradually without treatment as the patient develops. It is quite common for people here to say that they had a Derbyshire neck when they were young. The popular treatment in the female is to apply a string of artificial pearls or a black velvet neck band, and I was recently much interested to read in the British Medical Journal that the Abyssinian treatment of goitre is to tattoo a string of beads round the neck.

Besides the enlargement of the gland at the menstrual period I have seen the same occur during pregnancy, and decrease in size after childbirth.

When the goître enlarges to a further degree, the swelling is unsightly, the feeling of suffocation becomes more marked, especially in the recumbent posture. There is often associated anaemia, and the case demands treatment.

An inquiry into the residential situation of the patient is made, and the water supply and general hygienic surroundings ascertained. The water should be boiled and flavoured with lemon to make it palatable. Internally a mixture containing iodine and potassium iodide is given, and if there is anaemia, iron and arsenic also. Thyroid extract may be tried, commencing with $\frac{1}{2}$-grain doses every other day, and gradually increasing it up to 1 grain three times daily. But the effect on the pulse should be most care. fully watched. In my experience the result of treatment with thyroid extract has been very disappointing. If no diminution occurs with this treatment after a month's trial it is useless to continue. Locally the red iodide of mercury ointment has seemed to have some effect, the patient spreading it over the enlarged gland, and then sitting in bright sunshine or before a hot fire. It is very difficult not to blister the skin, however, and although good results are reported from India, its effect has not been very convincing in my own practice.

If such cases are watched for some time the swelling, instead of being uniform, becomes asymmetrical. The changes producing the asymmetrical enlargement are briefly as follows: The acini undergo great dilatation with colloid, the lining cubical cells become flattened, the intervening stroma is absorbed, and a small cyst is formed. This degenerative cystic formation generally causes the formation not of a single cyst but multiple small ones. Haemorrhage may take place in these, thus increasing their volume. Another cause of asymmetry is the growth of a definite encapsulated adenoma, which in its turn often becomes cystic. There may be one or many adenomata, and all stages of cystic degeneration of these may be seen in one gland. Again, asymmetrical enlargement is seen in carcinoma, primary or secondary, in sarcoma, and in endothelioma of the gland. Suppuration is yet another cause of asymmetricalenlargement, and is due to breaking down of new growths, especially sarcoma, to suppuration in a pre-existing cyst, either due to auto-infection or to the injudicious introduction of a septic needle. Metastatic pyogenic deposits have been seen in pyaemia and very rarely gummata and tuberculous granulomata.

If the parenchymatous goître is of long standing, other degenerative phenomena are common. Fibrous degeneration, with shrinking of the acini and blood vessels and great increase of the fibrous tissue stroma, may be found. This has been observed to lead to myxoedema, or at any rate patients with myxoedema sometimes give a history of having had previously an enlarged thyroid. Impregnation of the fibrous tissue stroma and colloid substance with lime salts is quite common, and true bone is said to have been observed. Colloid degeneration of the stroma itself is sometimes seen; and in such cases the walls of the blood vessels, becoming softened, may give way and a. sudden enlargement of the gland due to haemorrhage take place. Amyloid degeneration is occasionally found, the arteries being the chief seat of change, but circumscribed amyloid deposits have been also seen.

\section{Course.}

Many goittres, besides the deformity and slight dyspnoea, give rise to no other symptoms.

Dyspnoea, however, is often urgent, and depends on several factors. The gland being tightly bound round the trachea by the deep cervical fascia causes lateral pressure 